\title{
Existence of Solutions for Some Nonlinear Problems with Boundary Value Conditions
}

\author{
Dionicio Pastor Dallos Santos \\ Department of Mathematics, IME-USP, Cidade Universitária, 05508-090 São Paulo, SP, Brazil \\ Correspondence should be addressed to Dionicio Pastor Dallos Santos; dionicio@ime.usp.br \\ Received 7 June 2016; Revised 9 August 2016; Accepted 9 August 2016 \\ Academic Editor: Bingwen Liu
}

Copyright (c) 2016 Dionicio Pastor Dallos Santos. This is an open access article distributed under the Creative Commons Attribution License, which permits unrestricted use, distribution, and reproduction in any medium, provided the original work is properly cited.

\begin{abstract}
We study the existence of solutions for nonlinear boundary value problems $\left(\varphi\left(u^{\prime}\right)\right)^{\prime}=f\left(t, u, u^{\prime}\right), l\left(u, u^{\prime}\right)=0$, where $l\left(u, u^{\prime}\right)=0$ denotes the boundary conditions on a compact interval $[0, T], \varphi$ is a homeomorphism such that $\varphi(0)=0$, and $f:[0, T] \times \mathbb{R} \times \mathbb{R} \rightarrow$ $\mathbb{R}$ is a continuous function. All the contemplated boundary value problems are reduced to finding a fixed point for one operator defined on a space of functions, and Schauder fixed point theorem or Leray-Schauder degree is used.
\end{abstract}

\section{Introduction}

The purpose of this article is to obtain some existence results for nonlinear boundary value problems of the form

$$
\begin{aligned}
\left(\varphi\left(u^{\prime}\right)\right)^{\prime} & =f\left(t, u, u^{\prime}\right) \\
l\left(u, u^{\prime}\right) & =0
\end{aligned}
$$

where $l\left(u, u^{\prime}\right)=0$ denotes the Dirichlet or mixed boundary conditions on the interval $[0, T], \varphi$ is a bounded, singular, or classic homeomorphism such that $\varphi(0)=0, f:[0, T] \times$ $\mathbb{R} \times \mathbb{R} \rightarrow \mathbb{R}$ is a continuous function, and $T$ is a positive real number.

Recently, problem (1) in special cases, when $\varphi$ is an increasing homeomorphism from $(-a, a)$ to $\mathbb{R}$ such that $\varphi(0)=0$ and $l\left(u, u^{\prime}\right)=0$ denotes the periodic, Neumann, or Dirichlet boundary conditions, has been investigated by Bereanu and Mawhin in [1].

In [2], the authors have studied problem (1), where $\varphi$ : $\mathbb{R} \rightarrow(-a, a)(0<a \leq \infty)$ and $l\left(u, u^{\prime}\right)=0$ denotes the periodic boundary conditions. They obtained the existence of solutions by means of the Leray-Schauder degree theory. In particular, regular periodic problems with $\varphi$ - or $p$-Laplacian on the left hand side were considered by several authors; see, for example, del Pino et al. [3] or Yan [4].
In [5] Benevieri et al. proved an existence result for the periodic boundary value problem:

$$
\begin{aligned}
\left(\varphi\left(u^{\prime}\right)\right)^{\prime} & =f\left(t, u, u^{\prime}\right) \\
u(0) & =u(T), \\
u^{\prime}(0) & =u^{\prime}(T),
\end{aligned}
$$

assuming that $f:[0, T] \times \mathbb{R}^{n} \times \mathbb{R}^{n} \rightarrow \mathbb{R}^{n}$ is a Carathéodory function and $\varphi: \mathbb{R}^{n} \rightarrow \mathbb{R}^{n}$ is a homeomorphism between $\mathbb{R}^{n}$ and the open ball of $\mathbb{R}^{n}$ with center zero and radius 1 . They used a topological method: the properties of $\varphi$ and $f$ allowed applying the Leray-Schauder degree. The interest in this class of nonlinear operators $u \mapsto\left(\varphi\left(u^{\prime}\right)\right)^{\prime}$ is mainly due to the fact that they include the mean curvature operator:

$$
u \longmapsto \operatorname{div}\left(\frac{\nabla u}{\sqrt{1+|\nabla u|^{2}}}\right)
$$

The paper is organized as follows. In Section 2, we introduce some notations and preliminaries, which will be crucial in the proofs of our results. Section 3 is devoted to 
the study of existence of solutions for the Dirichlet problems with bounded homomorphisms:

$$
\begin{aligned}
\left(\varphi\left(u^{\prime}\right)\right)^{\prime} & =f\left(t, u, u^{\prime}\right), \\
u(0) & =0=u(T) .
\end{aligned}
$$

In particular, Bereanu and Mawhin in [6] proved the existence of at least one solution by means of the Leray-Schauder degree.

Theorem 1 (Bereanu and Mawhin). If the function $f$ satisfies the condition

$$
\begin{aligned}
& \exists c>0 \text { such that }|f(t, x, y)| \leq c<\frac{a}{2 T}, \\
& \forall(t, x, y) \in[0, T] \times \mathbb{R} \times \mathbb{R},
\end{aligned}
$$

the Dirichlet problem has at least one solution.

The main purpose of this section is an extension of the results obtained in the previous theorem. For this, we use topological methods based upon Leray-Schauder degree [7] and more general properties of the function $f$. In Section 4, we use the fixed point theorem of Schauder to show the existence of at least one solution for boundary value problems of the type

$$
\begin{aligned}
\left(\varphi\left(u^{\prime}\right)\right)^{\prime} & =f\left(t, u, u^{\prime}\right), \\
u(T) & =u(0)=u^{\prime}(T),
\end{aligned}
$$

where $\varphi:(-a, a) \rightarrow \mathbb{R}$ (we call it singular). Of course, a solution of $(6)$ is a function $u:[0, T] \rightarrow \mathbb{R}$ of class $C^{1}$ such that $\max _{[0, T]}\left|u^{\prime}(t)\right|<a$, satisfying the boundary conditions, and the function $\varphi\left(u^{\prime}\right)$ is continuously differentiable and $\left(\varphi\left(u^{\prime}(t)\right)\right)^{\prime}=f\left(t, u(t), u^{\prime}(t)\right)$ for all $t \in[0, T]$. In Section 5, for $u(T)=u^{\prime}(0)=u^{\prime}(T)$ boundary conditions and classic homeomorphisms $(\varphi: \mathbb{R} \rightarrow \mathbb{R})$, we investigate the existence of at least one solution using Leray-Schauder degree, where a solution of this problem is any function $u:[0, T] \rightarrow$ $\mathbb{R}$ of class $C^{1}$ such that $\varphi\left(u^{\prime}\right)$ is continuously differentiable, which satisfies the boundary conditions and $\left(\varphi\left(u^{\prime}(t)\right)\right)^{\prime}=$ $f\left(t, u(t), u^{\prime}(t)\right)$ for all $t \in[0, T]$. Such problems do not seem to have been studied in the literature. In the present paper generally we follow the ideas of Bereanu and Mawhin $[1,2,6,8]$.

\section{Notation and Preliminaries}

For fixed $T$, we denote the usual norm in $L^{1}=L^{1}([0, T], \mathbb{R})$ by $\|\cdot\|_{L^{1}}$. Let $C=C([0, T], \mathbb{R})$ denote the Banach space of continuous functions from $[0, T]$ into $\mathbb{R}$, endowed with the uniform norm $\|\cdot\|_{\infty}, C^{1}=C^{1}([0, T], \mathbb{R})$ the Banach space of continuously differentiable functions from $[0, T]$ into $\mathbb{R}$, equipped with the usual norm $\|u\|_{1}=\|u\|_{\infty}+\left\|u^{\prime}\right\|_{\infty}$, and $C_{0}^{1}$ the closed subspace of $C^{1}$ defined by $C_{0}^{1}=\left\{u \in C^{1}: u(T)=\right.$ $0=u(0)\}$.
We introduce the following applications:

The Nemytskii operator $N_{f}: C^{1} \rightarrow C$ :

$$
N_{f}(u)(t)=f\left(t, u(t), u^{\prime}(t)\right) \text {. }
$$

The integration operator $H: C \rightarrow C^{1}$ :

$$
H(u)(t)=\int_{0}^{t} u(s) d s
$$

The following continuous linear applications:

$$
\begin{aligned}
K: C & \longrightarrow C^{1}, \\
K(u)(t) & =-\int_{t}^{T} u(s) d s, \\
Q: C & \longrightarrow C, \\
Q(u)(t) & =\frac{1}{T} \int_{0}^{T} u(s) d s, \\
S: C & \longrightarrow C, \\
S(u)(t) & =u(T), \\
P: C & \longrightarrow C, \\
P(u)(t) & =u(0) .
\end{aligned}
$$

For $u \in C$, we write

$$
\begin{aligned}
& u_{m}=\min _{[0, T]} u, \\
& u_{M}=\max _{[0, T]} u, \\
& u^{+}=\max \{u, 0\}, \\
& u^{-}=\max \{-u, 0\} .
\end{aligned}
$$

The following lemma is an adaptation of a result of [1] to the case of a homeomorphism which is not defined everywhere. We present here the demonstration for better understanding of the development of our research.

Lemma 2. Let $B=\left\{h \in C:\|h\|_{\infty}<a / 2\right\}$. For each $h \in$ $B$, there exists a unique $Q_{\varphi}=Q_{\varphi}(h) \in \operatorname{Im}(h)$ (where $\operatorname{Im}(h)$ denotes the range of $h$ ) such that

$$
\int_{0}^{T} \varphi^{-1}\left(h(t)-Q_{\varphi}(h)\right) d t=0 .
$$

Moreover, the function $Q_{\varphi}: B \rightarrow \mathbb{R}$ is continuous and sends bounded sets into bounded sets.

Proof. Let $h \in B$. We define the continuous application $G_{h}$ : $\left[h_{m}, h_{M}\right] \rightarrow \mathbb{R}$ for

$$
G_{h}(s)=\int_{0}^{T} \varphi^{-1}(h(t)-s) d t .
$$


We now show that the equation

$$
G_{h}(s)=0
$$

has a unique solution $Q_{\varphi}(h)$. Let $r, s \in\left[h_{m}, h_{M}\right]$ be such that

$$
\begin{aligned}
& \int_{0}^{T} \varphi^{-1}(h(t)-r) d t=0 \\
& \int_{0}^{T} \varphi^{-1}(h(t)-s) d t=0
\end{aligned}
$$

that is,

$$
\int_{0}^{T} \varphi^{-1}(h(t)-r) d t=\int_{0}^{T} \varphi^{-1}(h(t)-s) d t .
$$

It follows that there exists $\tau \in[0, T]$ such that

$$
\varphi^{-1}(h(\tau)-r)=\varphi^{-1}(h(\tau)-s) .
$$

Using the injectivity of $\varphi^{-1}$ we deduce that $r=s$. Let us now show the existence. Because $\varphi^{-1}$ is strictly monotone and $\varphi^{-1}(0)=0$, we have that

$$
G_{h}\left(h_{m}\right) G_{h}\left(h_{M}\right) \leq 0 .
$$

It follows that there exists $s \in\left[h_{m}, h_{M}\right]$ such that $G_{h}(s)=0$. Consequently for each $h \in B$, (13) has a unique solution. Thus, we define the function $Q_{\varphi}: B \rightarrow \mathbb{R}$ such that

$$
\int_{0}^{T} \varphi^{-1}\left(h(t)-Q_{\varphi}(h)\right) d t=0 .
$$

On the other hand, because $h \in B$, we have that

$$
\left|Q_{\varphi}(h)\right| \leq\|h\|_{\infty}<\frac{a}{2}
$$

Therefore, the function $Q_{\varphi}$ sends bounded sets into bounded sets.

Finally, we show that $Q_{\varphi}$ is continuous on $B$. Let $\left(h_{n}\right)_{n} \subset C$ be a sequence such that $h_{n} \rightarrow h$ in $C$. Since the function $Q_{\varphi}$ sends bounded sets into bounded sets, then $\left(Q_{\varphi}\left(h_{n}\right)\right)_{n}$ is bounded. Hence, $\left(Q_{\varphi}\left(h_{n}\right)\right)_{n}$ is relatively compact. Without loss of generality, passing if necessary to a subsequence, we can assume that

$$
\lim _{n \rightarrow \infty} Q_{\varphi}\left(h_{n}\right)=\tilde{a},
$$

where for each $n \in \mathbb{N}$ we obtain

$$
\lim _{n \rightarrow \infty} \int_{0}^{T} \varphi^{-1}\left(h_{n}(t)-Q_{\varphi}\left(h_{n}\right)\right) d t=0 .
$$

Using the dominated convergence theorem, we deduce that

$$
\int_{0}^{T} \varphi^{-1}(h(t)-\widetilde{a}) d t=0,
$$

so we have that $Q_{\varphi}(h)=\tilde{a}$. Hence, the function $Q_{\varphi}$ is continuous.
The following extended homotopy invariance property of the Leray-Schauder degree can be found in [9].

Proposition 3. Let $X$ be a real Banach space, $V \subset[0,1] \times X$ be an open, bounded set, and $M$ be a completely continuous operator on $\bar{V}$ such that $x \neq M(\lambda, x)$ for each $(\lambda, x) \in \partial V$. Then the Leray-Schauder degree

$$
\operatorname{deg}_{L S}\left(I-M(\lambda, \cdot), V_{\lambda}, 0\right)
$$

is well defined and independent of $\lambda$ in $[0,1]$, where $V_{\lambda}$ is the open, bounded (possibly empty) set defined by $V_{\lambda}=\{x \in X$ : $(\lambda, x) \in V\}$.

\section{Dirichlet Problems with Bounded Homeomorphisms}

In this section we are interested in Dirichlet boundary value problems of the type

$$
\begin{aligned}
\left(\varphi\left(u^{\prime}\right)\right)^{\prime} & =f\left(t, u, u^{\prime}\right), \\
u(0) & =0=u(T),
\end{aligned}
$$

where $\varphi: \mathbb{R} \rightarrow(-a, a)$ is a homeomorphism, $\varphi(0)=0$, and $f:[0, T] \times \mathbb{R} \times \mathbb{R} \rightarrow \mathbb{R}$ is continuous. In order to apply LeraySchauder degree theory to show the existence of at least one solution of (24), we introduce, for $\lambda \in[0,1]$, the family of Dirichlet boundary value problems:

$$
\begin{gathered}
\left(\varphi\left(u^{\prime}\right)\right)^{\prime}=\lambda f\left(t, u, u^{\prime}\right), \\
u(0)=0=u(T) .
\end{gathered}
$$

Let

$$
\Omega=\left\{(\lambda, u) \in[0,1] \times C_{0}^{1}:\left\|\lambda H\left(N_{f}(u)\right)\right\|_{\infty}<\frac{a}{2}\right\} .
$$

Clearly $\Omega$ is an open set in $[0,1] \times C_{0}^{1}$ and is nonempty because $\{0\} \times C_{0}^{1} \subset \Omega$. Using Lemma 2, we can define the operator $M: \Omega \rightarrow C_{0}^{1}$ by

$$
\begin{aligned}
M & (\lambda, u) \\
& =H\left(\varphi^{-1}\left[\lambda H\left(N_{f}(u)\right)-Q_{\varphi}\left(\lambda H\left(N_{f}(u)\right)\right)\right]\right) .
\end{aligned}
$$

Here $\varphi^{-1}$ with an abuse of notation is understood as the operator $\varphi^{-1}: B_{a}(0) \subset C \rightarrow C$ defined by $\varphi^{-1}(v)(t)=$ $\varphi^{-1}(v(t))$. It is clear that $\varphi^{-1}$ is continuous and sends bounded sets into bounded sets.

When the boundary conditions are periodic or Neumann, an operator has been considered by Bereanu and Mawhin [6].

The following lemma plays a pivotal role in studying the solutions of problem (25).

Lemma 4. The operator $M$ is well defined and continuous. Moreover, if $(\lambda, u) \in \Omega$ is such that $M(\lambda, u)=u$, then $u$ is solution of (25). 
Proof. Let $(\lambda, u) \in \Omega$. We show that in fact $M(\lambda, u) \in C_{0}^{1}$. It is clear that

$$
\begin{aligned}
& (M(\lambda, u))^{\prime} \\
& \quad=\varphi^{-1}\left[\lambda H\left(N_{f}(u)\right)-Q_{\varphi}\left(\lambda H\left(N_{f}(u)\right)\right)\right],
\end{aligned}
$$

where the continuity of $M(\lambda, u)$ and $(M(\lambda, u))^{\prime}$ follows from the continuity of applications $H$ and $N_{f}$.

On the other hand using Lemma 2, we have

$$
M(\lambda, u)(0)=0=M(\lambda, u)(T) .
$$

Therefore $M(\Omega) \subset C_{0}^{1}$ and $M$ is well defined. The continuity of $M$ follows by the continuity of the operators which compose it $M$.

Now suppose that $(\lambda, u) \in \Omega$ is such that $M(\lambda, u)=u$. It follows from (27) that

$$
\begin{aligned}
u(t) & =M(\lambda, u)(t) \\
= & H\left(\varphi^{-1}\left[\lambda H\left(N_{f}(u)\right)-Q_{\varphi}\left(\lambda H\left(N_{f}(u)\right)\right)\right]\right)(t)
\end{aligned}
$$

for all $t \in[0, T]$. Differentiating (30), we obtain

$$
\begin{aligned}
u^{\prime}(t) & =\varphi^{-1}\left[\lambda H\left(N_{f}(u)\right)-Q_{\varphi}\left(\lambda H\left(N_{f}(u)\right)\right)\right](t) \\
& =\varphi^{-1}\left[\lambda H\left(N_{f}(u)\right)(t)-Q_{\varphi}\left(\lambda H\left(N_{f}(u)\right)\right)\right] .
\end{aligned}
$$

Applying $\varphi$ to both of its members we have that

$$
\varphi\left(u^{\prime}(t)\right)=\lambda H\left(N_{f}(u)\right)(t)-Q_{\varphi}\left(\lambda H\left(N_{f}(u)\right)\right) .
$$

Differentiating again, we deduce that

$$
\left(\varphi\left(u^{\prime}(t)\right)\right)^{\prime}=\lambda N_{f}(u)(t)
$$

for all $t \in[0, T]$. Thus, $u$ satisfies problem (25). This completes the proof.

Remark 5. Note that the reciprocal of Lemma 4 is not true because we cannot guarantee that $\| \lambda H\left(N_{f}(u) \|_{\infty}<a / 2\right.$ for every solution $u$ of (25).

In our main result, we need the following lemma to obtain the required a priori bounds for the possible fixed points of $M$.

Lemma 6. Assume that there exist $h \in C\left([0, T], \mathbb{R}^{+}\right)$and $n \in$ $C^{1}(\mathbb{R}, \mathbb{R})$ such that $\|h\|_{L^{1}}<a / 2, n(0)=0$,

$$
\begin{gathered}
\varphi(y) n^{\prime}(x) y \geq 0, \quad(t, x, y) \in[0, T] \times \mathbb{R} \times \mathbb{R}, \\
|f(t, x, y)| \leq f(t, x, y) n(x)+h(t)
\end{gathered}
$$

for all $(t, x, y) \in[0, T] \times \mathbb{R} \times \mathbb{R}$. If $(\lambda, u) \in \Omega$ is such that $M(\lambda, u)=u$, then

$$
\begin{aligned}
\left\|\lambda H\left(N_{f}(u)\right)\right\|_{\infty} & \leq\|h\|_{L^{1}}, \\
\left\|u^{\prime}\right\|_{\infty} & \leq L, \\
\|u\|_{1} & \leq L+L T,
\end{aligned}
$$

where $L=\max \left\{\left|\varphi^{-1}\left(-2\|h\|_{L^{1}}\right)\right|,\left|\varphi^{-1}\left(2\|h\|_{L^{1}}\right)\right|\right\}$.
Proof. Let $\lambda \neq 0$ and $(\lambda, u) \in \Omega$ be such that $M(\lambda, u)=u$. Using Lemma 4 , we have that $u$ is solution of (25), which implies that

$$
\begin{aligned}
\varphi\left(u^{\prime}\right) & =\lambda H\left(N_{f}(u)\right)-Q_{\varphi}\left(\lambda H\left(N_{f}(u)\right)\right), \\
u(0) & =0=u(T),
\end{aligned}
$$

where, for all $t \in[0, T]$, we obtain

$$
\begin{aligned}
\left|\lambda H\left(N_{f}(u)\right)(t)\right| \leq & \int_{0}^{T}\left|f\left(s, u(s), u^{\prime}(s)\right)\right| d s \\
\leq & \int_{0}^{T} f\left(s, u(s), u^{\prime}(s)\right) n(u(s)) d s \\
& +\int_{0}^{T} h(s) d s .
\end{aligned}
$$

On the other hand, using inequality (34) we have that

$$
-\int_{0}^{T} \varphi\left(u^{\prime}(t)\right) n^{\prime}(u(t)) u^{\prime}(t) d t \leq 0 .
$$

Using the integration by parts formula, the boundary conditions, and the fact that $n(0)=0$, we deduce that

$$
\begin{aligned}
& \int_{0}^{T}\left(\varphi\left(u^{\prime}(t)\right)\right)^{\prime} n(u(t)) d t \\
& \quad=-\int_{0}^{T} \varphi\left(u^{\prime}(t)\right) n^{\prime}(u(t)) u^{\prime}(t) d t \leq 0 .
\end{aligned}
$$

Since $\lambda \in(0,1]$ and $u$ is solution of $(25)$, it follows that

$$
\int_{0}^{T} f\left(t, u(t), u^{\prime}(t)\right) n(u(t)) d t \leq 0,
$$

and hence

$$
\left|\lambda H\left(N_{f}(u)\right)(t)\right| \leq\|h\|_{L^{1}} .
$$

On the other hand, since $Q_{\varphi}\left(\lambda H\left(N_{f}(u)\right)\right) \quad \epsilon$ $\operatorname{Im}\left(\lambda H\left(N_{f}(u)\right)\right)$, we get

$$
\left|\varphi\left(u^{\prime}(t)\right)\right| \leq 2\|h\|_{L^{1}}
$$

for all $t \in[0, T]$. It follows that

$$
\left\|\varphi\left(u^{\prime}\right)\right\|_{\infty} \leq 2\|h\|_{L^{1}},
$$

which implies that $\left\|u^{\prime}\right\|_{\infty} \leq L$, where $L=$ $\max \left\{\left|\varphi^{-1}\left(-2\|h\|_{L^{1}}\right)\right|,\left|\varphi^{-1}\left(2\|h\|_{L^{1}}\right)\right|\right\}$. Using again the boundary conditions, we have that

$$
|u(t)| \leq \int_{0}^{t}\left|u^{\prime}(s)\right| d s \leq \int_{0}^{T}\left|u^{\prime}(s)\right| d s \leq L T
$$

$$
(t \in[0, T]),
$$

and hence

$$
\|u\|_{1} \leq L+L T .
$$

Finally, if $u=M(0, u)$, then $u=0$, so the proof is complete. 
Let $\rho, \kappa \in \mathbb{R}$ be such that $\|h\|_{L^{1}}<\kappa<a / 2, \rho>L+L T$ and consider the set

$$
\begin{aligned}
V & =\left\{(\lambda, u) \in[0,1] \times C_{0}^{1}:\left\|\lambda H\left(N_{f}(u)\right)\right\|_{\infty}\right. \\
& \left.<\kappa,\|u\|_{1}<\rho\right\} .
\end{aligned}
$$

Since the set $\{0\} \times\left\{u \in C_{0}^{1}:\|u\|_{1}<\rho\right\} \subset V$, then we deduce that $V$ is nonempty. Moreover, it is clear that $V$ is open and bounded in $[0,1] \times C_{0}^{1}$ and $\bar{V} \subset \Omega$. On the other hand using an argument similar to the one introduced in the proof of Lemma 6 in [6], it is not difficult to see that $M: \bar{V} \rightarrow C_{0}^{1}$ is well defined and completely continuous and

$$
u \neq M(\lambda, u) \quad \forall(\lambda, u) \in \partial V .
$$

3.1. Existence Results. In this subsection, we present and prove our main result.

Theorem 7. If $f$ satisfies conditions of Lemma 6 , then problem (24) has at least one solution.

Proof. Let $M$ be the operator given by (27). Using Proposition 3 , we deduce that

$$
\begin{aligned}
& \operatorname{deg}_{\mathrm{LS}}\left(I-M(0, \cdot), V_{0}, 0\right) \\
& \quad=\operatorname{deg}_{\mathrm{LS}}\left(I-M(1, \cdot), V_{1}, 0\right),
\end{aligned}
$$

where $\operatorname{deg}_{\mathrm{LS}}\left(I-M(0, \cdot), V_{0}, 0\right)=\operatorname{deg}_{\mathrm{LS}}\left(I, B_{\rho}(0), 0\right)=1$. Thus, there exists $u \in V_{1}$ such that $M(1, u)=u$, which is a solution for (24).

Remark 8. Note that Theorem 7 is a generalization of Theorem 1.

Corollary 9. Assume that $\varphi$ is an increasing homomorphism. Let $h \in C\left([0, T], \mathbb{R}^{+}\right)$be such that

$$
\begin{aligned}
\|h\|_{L^{1}} & <\frac{a}{2}, \\
|f(t, x, y)| & \leq f(t, x, y) x+h(t)
\end{aligned}
$$

for all $x, y \in \mathbb{R}$ and $t \in[0, T]$. If $(\lambda, u) \in \Omega$ is such that $M(\lambda, u)=u$, then

$$
\begin{aligned}
\left\|\lambda H\left(N_{f}(u)\right)\right\|_{\infty} & \leq\|h\|_{L^{1}}, \\
\left\|u^{\prime}\right\|_{\infty} & \leq L, \\
\|u\|_{1} & \leq L+L T,
\end{aligned}
$$

where $L=\max \left\{\left|\varphi^{-1}\left(-2\|h\|_{L^{1}}\right)\right|,\left|\varphi^{-1}\left(2\|h\|_{L^{1}}\right)\right|\right\}$.

Proof. Since $\varphi$ is an increasing homomorphism we have that

$$
\varphi(y) y \geq 0
$$

for all $y \in \mathbb{R}$. Using Lemma 6 with $n(x)=x$ for all $x \in$ $\mathbb{R}$, we can obtain the conclusion of Corollary 9. The proof is achieved.
Theorem 10. If $f$ satisfies conditions of Corollary 9, then problem (24) has at least one solution.

Let us give now an application of Theorem 10 when $f$ is unbounded.

Example 11. Consider the Dirichlet problem

$$
\begin{aligned}
\left(\varphi\left(u^{\prime}\right)\right)^{\prime} & =u-2, \\
u(0) & =u(T)=0,
\end{aligned}
$$

where $\varphi(s)=s / \sqrt{1+s^{2}}$.

It is not difficult to verify that $\varphi: \mathbb{R} \rightarrow(-1,1)$ is an increasing homeomorphism and $f(t, x, y)=x-2$ is a continuous function such that

$$
\begin{aligned}
& |f(t, x, y)|=|x-2| \leq(x-2) x+4, \\
& (t, x, y) \in[0, T] \times \mathbb{R} \times \mathbb{R} .
\end{aligned}
$$

So, we can choose $h(t)=4$ and $T<1 / 8$ to see that Corollary 9 holds and so, using Theorem 10, we obtain that (53) has at least one solution.

\section{Problems with Singular Homeomorphisms and Three-Point Boundary Conditions}

In this section we study the existence of at least one solution for boundary value problems of the type

$$
\begin{aligned}
\left(\varphi\left(u^{\prime}\right)\right)^{\prime} & =f\left(t, u, u^{\prime}\right), \\
u(T) & =u(0)=u^{\prime}(T),
\end{aligned}
$$

where $\varphi:(-a, a) \rightarrow \mathbb{R}$ is a homeomorphism such that $\varphi(0)=$ 0 and $f:[0, T] \times \mathbb{R} \times \mathbb{R} \rightarrow \mathbb{R}$ is a continuous function.

In order to transform problem (55) to a fixed point problem we use a similar argument introduced in Lemma 2 for $h \in C$.

Lemma 12. $u \in C^{1}$ is a solution of (55) if and only if $u$ is a fixed point of the operator $M$ defined on $C^{1}$ by

$$
\begin{aligned}
M(u) & \\
= & \varphi^{-1}\left(-Q_{\varphi}\left(K\left(N_{f}(u)\right)\right)\right) \\
& +H\left(\varphi^{-1}\left[K\left(N_{f}(u)\right)-Q_{\varphi}\left(K\left(N_{f}(u)\right)\right)\right]\right) .
\end{aligned}
$$

Proof. If $u \in C^{1}$ is solution of (55), then

$$
\begin{aligned}
\left(\varphi\left(u^{\prime}(t)\right)\right)^{\prime} & =N_{f}(u)(t)=f\left(t, u(t), u^{\prime}(t)\right), \\
u(0) & =u(T), \\
u(0) & =u^{\prime}(T)
\end{aligned}
$$

for all $t \in[0, T]$. Applying $K$ to both members and using the fact that $u(0)=u^{\prime}(T)$, we deduce that

$$
\varphi\left(u^{\prime}(t)\right)=\varphi(u(0))+K\left(N_{f}(u)\right)(t) .
$$


By the inversion of $\varphi$ in (58), we have

$$
u^{\prime}(t)=\varphi^{-1}\left[K\left(N_{f}(u)\right)(t)+c\right]
$$

where $c=\varphi(u(0))$. Integrating from 0 to $t \in[0, T]$, we have that

$$
u(t)=u(0)+H\left(\varphi^{-1}\left[K\left(N_{f}(u)\right)+c\right]\right)(t) .
$$

Because $u(0)=u(T)$, then

$$
\int_{0}^{T} \varphi^{-1}\left[K\left(N_{f}(u)\right)(t)+c\right] d t=0
$$

Using an argument similar to the one introduced in Lemma 2, it follows that $c=-Q_{\varphi}\left(K\left(N_{f}(u)\right)\right)$. Hence,

$$
\begin{aligned}
u= & \varphi^{-1}\left(-Q_{\varphi}\left(K\left(N_{f}(u)\right)\right)\right) \\
& +H\left(\varphi^{-1}\left[K\left(N_{f}(u)\right)-Q_{\varphi}\left(K\left(N_{f}(u)\right)\right)\right]\right) .
\end{aligned}
$$

Let $u \in C^{1}$ be such that $u=M(u)$. Then

$u(t)$

$$
\begin{aligned}
= & \varphi^{-1}\left(-Q_{\varphi}\left(K\left(N_{f}(u)\right)\right)\right) \\
& +H\left(\varphi^{-1}\left[K\left(N_{f}(u)\right)-Q_{\varphi}\left(K\left(N_{f}(u)\right)\right)\right]\right)(t)
\end{aligned}
$$

for all $t \in[0, T]$. Since $\int_{0}^{T} \varphi^{-1}\left[K\left(N_{f}(u)\right)(t)-\right.$ $\left.Q_{\varphi}\left(K\left(N_{f}(u)\right)\right)\right] d t=0$, therefore, we have that $u(0)=u(T)$. Differentiating (63), we obtain that

$$
u^{\prime}(t)=\varphi^{-1}\left[K\left(N_{f}(u)\right)-Q_{\varphi}\left(K\left(N_{f}(u)\right)\right)\right](t) .
$$

In particular,

$$
\begin{aligned}
u^{\prime}(T) & =\varphi^{-1}\left(0-Q_{\varphi}\left(K\left(N_{f}(u)\right)\right)\right) \\
& =\varphi^{-1}\left(-Q_{\varphi}\left(K\left(N_{f}(u)\right)\right)\right)=u(0) .
\end{aligned}
$$

Applying $\varphi$ to both members and differentiating again, we deduce that

$$
\begin{aligned}
\left(\varphi\left(u^{\prime}(t)\right)\right)^{\prime} & =N_{f}(u)(t), \\
u(0) & =u(T), \\
u(0) & =u^{\prime}(T)
\end{aligned}
$$

for all $t \in[0, T]$. This completes the proof.

Lemma 13. The operator $M: C^{1} \rightarrow C^{1}$ is completely continuous.

Proof. Let $\Lambda \subset C^{1}$ be a bounded set. Then, if $u \in \Lambda$, there exists a constant $\rho>0$ such that

$$
\|u\|_{1} \leq \rho
$$

Next, we show that $\overline{M(\Lambda)} \subset C^{1}$ is a compact set. Let $\left(v_{n}\right)_{n}$ be a sequence in $M(\Lambda)$, and let $\left(u_{n}\right)_{n}$ be a sequence in $\Lambda$ such that $v_{n}=M\left(u_{n}\right)$. Using (67), we have that there exists a constant $L>0$ such that, for all $n \in \mathbb{N}$,

$$
\left\|N_{f}\left(u_{n}\right)\right\|_{\infty} \leq L
$$

which implies that

$$
\left\|K\left(N_{f}\left(u_{n}\right)\right)-Q_{\varphi}\left(K\left(N_{f}\left(u_{n}\right)\right)\right)\right\|_{\infty} \leq 2 L T .
$$

Hence the sequence $\left(K\left(N_{f}\left(u_{n}\right)\right)-Q_{\varphi}\left(K\left(N_{f}\left(u_{n}\right)\right)\right)\right)_{n}$ is bounded in $C$. Moreover, for $t, t_{1} \in[0, T]$ and for all $n \in \mathbb{N}$, we have that

$$
\begin{aligned}
& \mid K\left(N_{f}\left(u_{n}\right)\right)(t)-Q_{\varphi}\left(K\left(N_{f}\left(u_{n}\right)\right)\right) \\
& \quad-K\left(N_{f}\left(u_{n}\right)\right)\left(t_{1}\right)+Q_{\varphi}\left(K\left(N_{f}\left(u_{n}\right)\right)\right) \mid \\
& \quad \leq \mid-\int_{t}^{T} f\left(s, u_{n}(s), u_{n}^{\prime}(s)\right) d s \\
& \quad+\int_{t_{1}}^{T} f\left(s, u_{n}(s), u_{n}^{\prime}(s)\right) d s \mid \\
& \quad \leq\left|\int_{t_{1}}^{t} f\left(s, u_{n}(s), u_{n}^{\prime}(s)\right) d s\right| \leq L\left|t-t_{1}\right|,
\end{aligned}
$$

which implies that $\left(K\left(N_{f}\left(u_{n}\right)\right)-Q_{\varphi}\left(K\left(N_{f}\left(u_{n}\right)\right)\right)\right)_{n}$ is equicontinuous. Thus, by the Arzelà-Ascoli theorem there is a subsequence of $\left(K\left(N_{f}\left(u_{n}\right)\right)-Q_{\varphi}\left(K\left(N_{f}\left(u_{n}\right)\right)\right)\right)_{n}$, which we call $\left(K\left(N_{f}\left(u_{n_{j}}\right)\right)-Q_{\varphi}\left(K\left(N_{f}\left(u_{n_{j}}\right)\right)\right)\right)_{j}$, which is convergent in $C$. Using the fact that $\varphi^{-1}: C \rightarrow B_{a}(0) \subset C$ is continuous, it follows from

$$
\begin{aligned}
& M\left(u_{n_{j}}\right)^{\prime} \\
& \quad=\varphi^{-1}\left[K\left(N_{f}\left(u_{n_{j}}\right)\right)-Q_{\varphi}\left(K\left(N_{f}\left(u_{n_{j}}\right)\right)\right)\right]
\end{aligned}
$$

that the sequence $\left(M\left(u_{n_{j}}\right)^{\prime}\right)_{j}$ is convergent in $C$. Then, passing to a subsequence if necessary, we obtain that $\left(v_{n_{j}}\right)_{j}=$ $\left(M\left(u_{n_{j}}\right)\right)_{j}$ is convergent in $C^{1}$. Finally, let $\left(v_{n}\right)_{n}$ be a sequence in $\overline{M(\Lambda)}$. Let $\left(z_{n}\right)_{n} \subseteq M(\Lambda)$ be such that

$$
\lim _{n \rightarrow \infty}\left\|z_{n}-v_{n}\right\|_{1}=0
$$

Let $\left(z_{n_{j}}\right)_{j}$ be a subsequence of $\left(z_{n}\right)_{n}$ such that it converges to $z$. It follows that $z \in \overline{M(\Lambda)}$ and $\left(v_{n_{j}}\right)_{j}$ converge to $z$. This concludes the proof.

The next result is based on Schauder's fixed point theorem.

Theorem 14. Let $f:[0, T] \times \mathbb{R} \times \mathbb{R} \rightarrow \mathbb{R}$ be continuous. Then (55) has at least one solution. 
Proof. Let $u \in C^{1}$. Then

$M(u)$

$$
\begin{aligned}
= & \varphi^{-1}\left(-Q_{\varphi}\left(K\left(N_{f}(u)\right)\right)\right) \\
& +H\left(\varphi^{-1}\left[K\left(N_{f}(u)\right)-Q_{\varphi}\left(K\left(N_{f}(u)\right)\right)\right]\right),
\end{aligned}
$$

where

$$
\begin{gathered}
M(u)(0)=\varphi^{-1}\left(-Q_{\varphi}\left(K\left(N_{f}(u)\right)\right)\right)=M(u)(T), \\
M(u)^{\prime}(T)=\varphi^{-1}\left(-Q_{\varphi}\left(K\left(N_{f}(u)\right)\right)\right)=M(u)(0) .
\end{gathered}
$$

Moreover,

$$
\begin{aligned}
& \left\|M(u)^{\prime}\right\|_{\infty} \\
& \quad=\left\|\varphi^{-1}\left[K\left(N_{f}(u)\right)-Q_{\varphi}\left(K\left(N_{f}(u)\right)\right)\right]\right\|_{\infty}<a, \\
& \|M(u)\|_{\infty}<a+a T .
\end{aligned}
$$

Hence,

$$
\begin{aligned}
\|M(u)\|_{1} & =\|M(u)\|_{\infty}+\left\|M(u)^{\prime}\right\|_{\infty}<a+a T+a \\
& =2 a+a T .
\end{aligned}
$$

Because the operator $M$ is completely continuous and bounded, we can use Schauder's fixed point theorem to deduce the existence of at least one fixed point. This, in turn, implies that problem (55) has at least one solution. The proof is complete.

\section{Problems with Classic Homeomorphisms and Three-Point Boundary Conditions}

We finally consider boundary value problems of the form

$$
\begin{aligned}
\left(\varphi\left(u^{\prime}\right)\right)^{\prime} & =f\left(t, u, u^{\prime}\right), \\
u(T) & =u^{\prime}(0)=u^{\prime}(T),
\end{aligned}
$$

where $\varphi: \mathbb{R} \rightarrow \mathbb{R}$ is a homeomorphism such that $\varphi(0)=0$ and $f:[0, T] \times \mathbb{R} \times \mathbb{R} \rightarrow \mathbb{R}$ is a continuous function. We remember that a solution of this problem is any function $u:[0, T] \rightarrow \mathbb{R}$ of class $C^{1}$ such that $\varphi\left(u^{\prime}\right)$ is continuously differentiable, satisfying the boundary conditions and $\left(\varphi\left(u^{\prime}(t)\right)\right)^{\prime}=f\left(t, u(t), u^{\prime}(t)\right)$ for all $t \in[0, T]$.

Let us consider the operator

$$
\begin{aligned}
& M_{1}: C^{1} \longrightarrow C^{1}, \\
& u \longmapsto S(u)+Q\left(N_{f}(u)\right) \\
& \quad+K\left(\varphi^{-1}\left[H\left(N_{f}(u)-Q\left(N_{f}(u)\right)\right)+\varphi(S(u))\right]\right) .
\end{aligned}
$$

Analogously to Section 3 , here $\varphi^{-1}$ is understood as the operator $\varphi^{-1}: C \rightarrow C$ defined for $\varphi^{-1}(v)(t)=\varphi^{-1}(v(t))$. It is clear that $\varphi^{-1}$ is continuous and sends bounded sets into bounded sets.
Lemma 15. $u \in C^{1}$ is a solution of (77) if and only if $u$ is a fixed point of the operator $M_{1}$.

Proof. Let $u \in C^{1}$, and we have the following equivalences:

$$
\begin{aligned}
& \left(\varphi\left(u^{\prime}\right)\right)^{\prime}=N_{f}(u), \\
& u^{\prime}(T)=u^{\prime}(0), \\
& u^{\prime}(0)=u(T) \\
& \Longleftrightarrow\left(\varphi\left(u^{\prime}\right)\right)^{\prime}=N_{f}(u)-Q\left(N_{f}(u)\right), \\
& \quad Q\left(N_{f}(u)\right)=0, u^{\prime}(0)=u(T) \\
& \Longleftrightarrow \varphi\left(u^{\prime}\right)=H\left(N_{f}(u)-Q\left(N_{f}(u)\right)\right)+\varphi\left(u^{\prime}(0)\right), \\
& \quad Q\left(N_{f}(u)\right)=0, u^{\prime}(0)=u(T) \\
& \left.+\varphi\left(u^{\prime}(0)\right)\right], \quad Q\left(N_{f}(u)\right)=0, u^{\prime}(0)=u(T) \\
& +K\left(\varphi^{-1}\left[H\left(N_{f}(u)-Q\left(N_{f}(u)\right)\right)+\varphi(S(u))\right]\right) . \\
& \left.\left.+\varphi\left(u^{\prime}(0)\right)\right]\right), \quad Q\left(N_{f}(u)\right)=0, u^{\prime}(0)=u(T) \\
& \Longleftrightarrow u=u(T)+Q\left(N_{f}(u)\right) \\
& \left.\left.\qquad u\left(N_{f}(u)-Q\left(N_{f}(u)\right)\right)+\varphi(u(T))\right]\right)
\end{aligned}
$$

Remark 16. Note that $u^{\prime}(T)=u^{\prime}(0) \Leftrightarrow Q\left(N_{f}(u)\right)=0$.

Using an argument similar to the one introduced in Lemma 13 , it is easy to see that $M_{1}: C^{1} \rightarrow C^{1}$ is completely continuous.

In order to apply Leray-Schauder degree to the operator $M_{1}$, we introduced a family of problems depending on parameter $\lambda$. We remember that to each continuous function $f:[0, T] \times \mathbb{R} \times \mathbb{R} \rightarrow \mathbb{R}$ we associate its Nemytskii operator $N_{f}: C^{1} \rightarrow C$ defined by

$$
N_{f}(u)(t)=f\left(t, u(t), u^{\prime}(t)\right)
$$

For $\lambda \in[0,1]$, we consider the family of boundary value problems:

$$
\begin{aligned}
\left(\varphi\left(u^{\prime}\right)\right)^{\prime} & =\lambda N_{f}(u)+(1-\lambda) Q\left(N_{f}(u)\right), \\
u(T) & =u^{\prime}(0)=u^{\prime}(T) .
\end{aligned}
$$


Notice that (81) coincides with (77) for $\lambda=1$. So, for each $\lambda \in[0,1]$, the operator associated with (81) by Lemma 15 is the operator $M(\lambda, \cdot)$, where $M$ is defined on $[0,1] \times C^{1}$ by

$$
\begin{aligned}
& M(\lambda, u)=S(u)+Q\left(N_{f}(u)\right) \\
& \quad+K\left(\varphi ^ { - 1 } \left[\lambda H\left(N_{f}(u)-Q\left(N_{f}(u)\right)\right)\right.\right. \\
& \quad+\varphi(S(u))]) .
\end{aligned}
$$

Using the same arguments as in the proof of Lemma 13 we show that the operator $M$ is completely continuous. Moreover, using the same reasoning as above, system (81) (see Lemma 15) is equivalent to the problem

$$
u=M(\lambda, u) .
$$

5.1. Existence Results. In this subsection, we present and prove our main results. These results are inspired by works of Bereanu and Mawhin [6] and Manásevich and Mawhin [10]. We denote by $\operatorname{deg}_{B}$ the Brouwer degree and by $\operatorname{deg}_{L S}$ the Leray-Schauder degree and define the mapping $G: \mathbb{R}^{2} \rightarrow \mathbb{R}^{2}$ by

$$
\begin{aligned}
& G: \mathbb{R}^{2} \longrightarrow \mathbb{R}^{2} \\
&(a, b) \longmapsto\left(a T+b T^{2}-b T\right. \\
&\left.-\frac{1}{T} \int_{0}^{T} f(t, a+b t, b) d t, b-a-b T\right) .
\end{aligned}
$$

Theorem 17. Assume that $\Omega$ is an open bounded set in $C^{1}$ such that the following conditions hold:

(1) For each $\lambda \in(0,1)$ problem

$$
\begin{aligned}
\left(\varphi\left(u^{\prime}\right)\right)^{\prime} & =\lambda N_{f}(u), \\
u(T) & =u^{\prime}(0)=u^{\prime}(T),
\end{aligned}
$$

has no solution on $\partial \Omega$.

(2) The equation

$$
G(a, b)=(0,0)
$$

has no solution on $\partial \Omega \cap \mathbb{R}^{2}$, where we consider the natural identification $(a, b) \approx a+b t$ of $\mathbb{R}^{2}$ with related functions in $C^{1}$.

(3) The Brouwer degree

$$
\operatorname{deg}_{B}\left(G, \Omega \cap \mathbb{R}^{2}, 0\right) \neq 0 .
$$

Then problem (77) has a solution.

Proof. Let $\lambda \in(0,1]$. If $u$ is a solution of $(85)$, then $Q\left(N_{f}(u)\right)=0$; hence $u$ is a solution of problem (81). On the other hand, for $\lambda \in(0,1]$, if $u$ is a solution of $(81)$ and because

$$
Q\left(\lambda N_{f}(u)+(1-\lambda) Q\left(N_{f}(u)\right)\right)=Q\left(N_{f}(u)\right)
$$

we have $Q\left(N_{f}(u)\right)=0$; then $u$ is a solution of (85). It follows that, for $\lambda \in(0,1]$, problems (81) and (85) have the same solutions. We assume that, for $\lambda=1,(81)$ does not have a solution on $\partial \Omega$ since otherwise we are done with proof. It follows that $(81)$ has no solutions for $(\lambda, u) \in(0,1] \times \partial \Omega$. If $\lambda=0$, then (81) is equivalent to the problem

$$
\begin{aligned}
\left(\varphi\left(u^{\prime}\right)\right)^{\prime} & =Q\left(N_{f}(u)\right), \\
u(T) & =u^{\prime}(0)=u^{\prime}(T),
\end{aligned}
$$

and thus, if $u$ is a solution of (89), we must have

$$
\int_{0}^{T} f\left(t, u(t), u^{\prime}(t)\right) d t=0 .
$$

Moreover, $u$ is a function of the form $u(t)=a+b t, a=b-b T$. Thus, by (90)

$$
\int_{0}^{T} f(t, a+b t, b) d t=0,
$$

which, together with hypothesis (2), implies that $u=b-b T+$ $t b \notin \partial \Omega$. Thus we have proved that (81) has no solution in $\partial \Omega$ for all $\lambda \in[0,1]$. Then we have that, for each $\lambda \in[0,1]$, the Leray-Schauder degree $\operatorname{deg}_{\mathrm{LS}}(I-M(\lambda, \cdot), \Omega, 0)$ is well defined, and by the homotopy invariance, one has

$$
\operatorname{deg}_{\mathrm{LS}}(I-M(0, \cdot), \Omega, 0)=\operatorname{deg}_{\mathrm{LS}}(I-M(1, \cdot), \Omega, 0) .
$$

On the other hand, we have that

$$
\begin{aligned}
& \operatorname{deg}_{\mathrm{LS}}(I-M(0, \cdot), \Omega, 0) \\
& \quad=\operatorname{deg}_{\mathrm{LS}}\left(I-\left(S+Q N_{f}+K S\right), \Omega, 0\right) .
\end{aligned}
$$

But the range of the mapping

$$
u \longmapsto S(u)+Q\left(N_{f}(u)\right)+K(S(u))
$$

is contained in the subspace of related functions, isomorphic to $\mathbb{R}^{2}$. Thus, using a reduction property of Leray-Schauder degree $[7,11]$,

$$
\begin{aligned}
& \operatorname{deg}_{\mathrm{LS}}\left(I-\left(S+Q N_{f}+K S\right), \Omega, 0\right) \\
& \quad=\operatorname{deg}_{\mathrm{B}}\left(I-\left(S+Q N_{f}+K S\right) \mid \frac{}{\Omega \cap \mathbb{R}^{2}}, \Omega \cap \mathbb{R}^{2}, 0\right) \\
& \quad=\operatorname{deg}_{\mathrm{B}}\left(G, \Omega \cap \mathbb{R}^{2}, 0\right) \neq 0 .
\end{aligned}
$$

Then, $\operatorname{deg}_{\mathrm{LS}}(I-M(1, \cdot), \Omega, 0) \neq 0$, where $I$ denotes the unit operator. Hence, there exists $u \in \Omega$ such that $M_{1}(u)=u$, which is a solution for (77).

The following result gives a priori bounds for the possible solutions of (85) and adapts a technique introduced by Ward Jr. [12].

Theorem 18. Assume that $f$ satisfies the following conditions:

(1) There exists $c \in C$ such that

$$
f(t, x, y) \geq c(t)
$$

for all $(t, x, y) \in[0, T] \times \mathbb{R} \times \mathbb{R}$. 
(2) There exists $M_{1}<M_{2}$ such that, for all $u \in C^{1}$,

$$
\begin{aligned}
& \int_{0}^{T} f\left(t, u(t), u^{\prime}(t)\right) d t \neq 0 \quad \text { if } u_{m}^{\prime} \geq M_{2} \\
& \int_{0}^{T} f\left(t, u(t), u^{\prime}(t)\right) d t \neq 0 \quad \text { if } u_{M}^{\prime} \leq M_{1} .
\end{aligned}
$$

If $(\lambda, u) \in(0,1) \times C^{1}$ is such that $u$ is solution of $(85)$, then

$$
\|u\|_{1}<r(2+T)
$$

where

$$
\begin{aligned}
r & \\
\quad & =\max \left\{\left|\varphi^{-1}\left(L+2\left\|c^{-}\right\|_{L^{1}}\right)\right|,\left|\varphi^{-1}\left(-L-2\left\|c^{-}\right\|_{L^{1}}\right)\right|\right\}, \\
L & =\max \left\{\left|\varphi\left(M_{2}\right)\right|,\left|\varphi\left(M_{1}\right)\right|\right\} .
\end{aligned}
$$

Proof. Let $(\lambda, u) \in(0,1) \times C^{1}$ be such that $u$ is a solution of (85). Then, for all $t \in[0, T]$,

$$
\begin{aligned}
\left(\varphi\left(u^{\prime}(t)\right)\right)^{\prime} & =\lambda N_{f}(u)(t), \\
u^{\prime}(0) & =u^{\prime}(T)=u(T), \\
\int_{0}^{T} f\left(t, u(t), u^{\prime}(t)\right) d t & =0 .
\end{aligned}
$$

Using hypothesis (2), we have that

$$
\begin{aligned}
& u_{m}^{\prime}<M_{2}, \\
& u_{M}^{\prime}>M_{1} .
\end{aligned}
$$

It follows that there exists $\omega \in[0, T]$ such that $M_{1}<u^{\prime}(\omega)<$ $M_{2}$ and

$$
\int_{\omega}^{t}\left(\varphi\left(u^{\prime}(s)\right)\right)^{\prime} d s=\lambda \int_{\omega}^{t} N_{f}(u)(s) d s,
$$

which implies that

$$
\begin{aligned}
\left|\varphi\left(u^{\prime}(t)\right)\right| \leq & \left|\varphi\left(u^{\prime}(\omega)\right)\right| \\
& +\int_{0}^{T}\left|f\left(s, u(s), u^{\prime}(s)\right)\right| d s,
\end{aligned}
$$

where

$$
\begin{aligned}
& \int_{0}^{T}\left|f\left(s, u(s), u^{\prime}(s)\right)\right| d s \\
& \quad \leq \int_{0}^{T} f\left(s, u(s), u^{\prime}(s)\right) d s+2 \int_{0}^{T} c^{-}(s) d s .
\end{aligned}
$$

Hence,

$$
\left|\varphi\left(u^{\prime}(t)\right)\right|<L+2\left\|c^{-}\right\|_{L^{1}}
$$

where $L=\max \left\{\left|\varphi\left(M_{2}\right)\right|,\left|\varphi\left(M_{1}\right)\right|\right\}$ and $t \in[0, T]$. It follows that

$$
\left\|u^{\prime}\right\|_{\infty}<r,
$$

where $r=\max \left\{\left|\varphi^{-1}\left(L+2\left\|c^{-}\right\|_{L^{1}}\right)\right|,\left|\varphi^{-1}\left(-L-2\left\|c^{-}\right\|_{L^{1}}\right)\right|\right\}$. Because $u \in C^{1}$ is such that $u^{\prime}(0)=u(T)$ we have that

$$
|u(t)| \leq|u(T)|+\int_{0}^{T}\left|u^{\prime}(s)\right| d s<r+r T
$$

$$
(t \in[0, T]),
$$

and hence $\|u\|_{1}=\|u\|_{\infty}+\left\|u^{\prime}\right\|_{\infty}<r+r T+r=r(2+T)$. This proves the theorem.

Now we show the existence of at least one solution for problem (77) by means of Leray-Schauder degree.

Theorem 19. Let $f$ be continuous and let it satisfy conditions (1) and (2) of Theorem 18. Assume that the following conditions hold for some $\rho \geq r(2+T)$ :

(1) The equation

$$
G(a, b)=(0,0)
$$

has no solution on $\partial B_{\rho}(0) \cap \mathbb{R}^{2}$, where we consider the natural identification $(a, b) \approx a+b t$ of $\mathbb{R}^{2}$ with related functions in $C^{1}$.

(2) The Brouwer degree

$$
\operatorname{deg}_{B}\left(G, B_{\rho}(0) \cap \mathbb{R}^{2}, 0\right) \neq 0 .
$$

Then problem (77) has a solution.

Proof. Let $(\lambda, u) \in(0,1) \times C^{1}$ be such that $u$ is a solution of (85). Using Theorem 18, we have

$$
\|u\|_{1}=\|u\|_{\infty}+\left\|u^{\prime}\right\|_{\infty}<r+r T+r=r(2+T),
$$

where $r=\max \left\{\left|\varphi^{-1}\left(L+2\left\|c^{-}\right\|_{L^{1}}\right)\right|,\left|\varphi^{-1}\left(-L-2\left\|c^{-}\right\|_{L^{1}}\right)\right|\right\}$. Thus the conditions of Theorem 17 are satisfied with $\Omega=B_{\rho}(0)$, where $B_{\rho}(0)$ is the open ball in $C^{1}$ center 0 and radius $\rho$. This concludes the proof.

Let us give now an application of Theorem 19.

Example 20. Let us consider the problem

$$
\begin{aligned}
\left(\left(u^{\prime}\right)^{3}\right)^{\prime} & =\frac{e^{u^{\prime}}}{2}-1, \\
u(T) & =u^{\prime}(0)=u^{\prime}(T) .
\end{aligned}
$$

Let $M_{1}=-1$ and $M_{2}=1$. If we suppose that $u_{m}^{\prime} \geq M_{2}$ and $u_{M}^{\prime} \leq M_{1}$, then

$$
\int_{0}^{T}\left(\frac{e^{u^{\prime}(t)}}{2}-1\right) d t \geq\left(\frac{e^{M_{2}}}{2}-1\right) T>0,
$$

$$
\int_{0}^{T}\left(\frac{e^{u^{\prime}(t)}}{2}-1\right) d t \leq\left(\frac{e^{M_{1}}}{2}-1\right) T<0 .
$$


On the other hand, if we choose $\rho \geq(1+2 T)^{1 / 3}(2+T)$ and $c(t)=-1$ for all $t \in[0, T]$, we have that the equation

$$
\begin{aligned}
& G(a, b)=\left(a T+b T^{2}-b T\right. \\
& \left.-\frac{1}{T} \int_{0}^{T} f(t, a+b t, b) d t, b-a-b T\right)=(0,0) \\
& =\left(a T+b T^{2}-b T-\frac{1}{T} \int_{0}^{T}\left(\frac{e^{b}}{2}-1\right) d t, b-a\right. \\
& -b T)=(0,0)=\left(a T+b T^{2}-b T-\frac{e^{b}}{2}+1, b-a\right. \\
& -b T)=(0,0)
\end{aligned}
$$

has no solution on $\partial B_{\rho}(0) \cap \mathbb{R}^{2}$. Then we have that the Brouwer degree $\operatorname{deg}_{\mathrm{B}}\left(G, B_{\rho}(0) \cap \mathbb{R}^{2},(0,0)\right)$ is well defined and, by the properties of that degree, we have that

$$
\begin{aligned}
& \operatorname{deg}_{\mathrm{B}}\left(G, B_{\rho}(0) \cap \mathbb{R}^{2},(0,0)\right)=\sum_{x \in G^{-1}(0,0)} \operatorname{sgn} J_{G}(x) \\
& \quad \neq 0,
\end{aligned}
$$

where $(0,0)$ is a regular value of $G$ and $J_{G}(x)=\operatorname{det} G^{\prime}(x)$ is the Jacobian of $G$ at $x$. So, using Theorem 19, we obtain that the boundary value problem (111) has at least one solution.

\section{Competing Interests}

The author declares that there is no conflict of interests regarding the publication of this article.

\section{Acknowledgments}

This research was supported by CAPES and CNPq/Brazil. The author would like to thank Dr. Pierluigi Benevieri for his kind advice and for the constructive revision of this paper.

\section{References}

[1] C. Bereanu and J. Mawhin, "Existence and multiplicity results for some nonlinear problems with singular $\varphi$-laplacian," Journal of Differential Equations, vol. 243, no. 2, pp. 536-557, 2007.

[2] C. Bereanu and J. Mawhin, "Periodic solutions of nonlinear perturbations of $\varphi$," Nonlinear Analysis: Theory, Methods \& Applications, vol. 68, no. 6, pp. 1668-1681, 2008.

[3] M. A. del Pino, R. F. Manásevich, and A. E. Murúa, "Existence and multiplicity of solutions with prescribed period for a second order quasilinear O.D.E.", Nonlinear Analysis: Theory, Methods \& Applications, vol. 18, no. 1, pp. 79-92, 1992.

[4] P. Yan, "Nonresonance for one-dimensional $p$-Laplacian with regular restoring," Journal of Mathematical Analysis and Applications, vol. 285, no. 1, pp. 141-154, 2003.

[5] P. Benevieri, J. M. do Ó, and E. S. de Medeiros, "Periodic solutions for nonlinear systems with mean curvature-like operators," Nonlinear Analysis: Theory, Methods \& Applications, vol. 65, no. 7, pp. 1462-1475, 2006.
[6] C. Bereanu and J. Mawhin, "Boundary-value problems with non-surjective $\varphi$ - laplacian and one-sided bounded nonlinearity," Advances Differential Equations, vol. 11, no. 1, pp. 35-60, 2006.

[7] J. Mawhin, Topological Degree Methods in Nonlinear Boundary Value Problems, vol. 40 of CBMS Series, American Mathematical Society, Providence, RI, USA, 1979.

[8] C. Bereanu and J. Mawhin, "Boundary value problems for some nonlinear systems with singular $\phi$-laplacian," Journal of Fixed Point Theory and Applications, vol. 4, no. 1, pp. 57-75, 2008.

[9] J. Mawhin, "Leray-Schauder degree: a half century of extensions and applications," Topological Methods in Nonlinear Analysis, vol. 14, no. 2, pp. 195-228, 1999.

[10] R. Manásevich and J. Mawhin, "Periodic solutions for nonlinear systems with $p$-Laplacian-like operators," Journal of Differential Equations, vol. 145, no. 2, pp. 367-393, 1998.

[11] K. Deimling, Nonlinear Functional Analysis, Springer, Berlin, Germany, 1985.

[12] J. R. Ward Jr., "Asymptotic conditions for periodic solutions of ordinary differential equations," Proceedings of the American Mathematical Society, vol. 81, no. 3, pp. 415-420, 1981. 


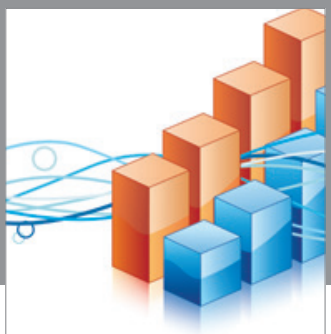

Advances in

Operations Research

vatem alat4

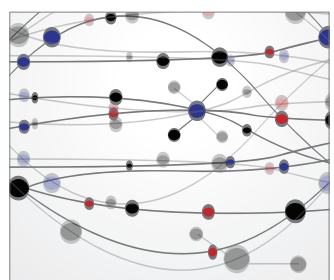

\section{The Scientific} World Journal
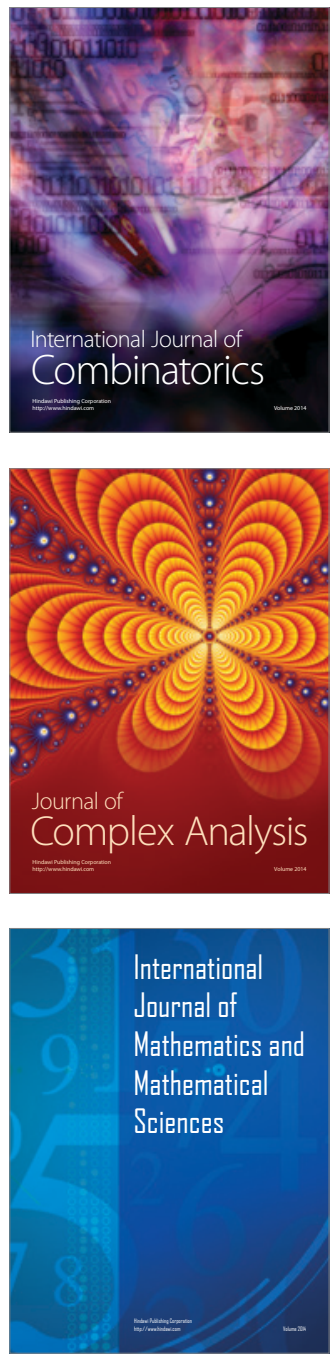
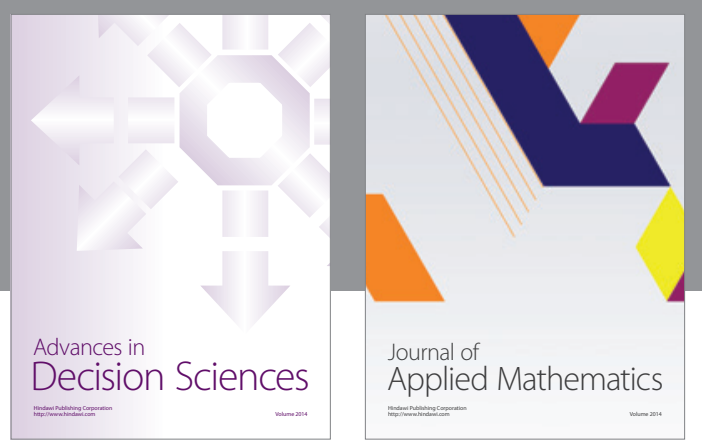

Algebra

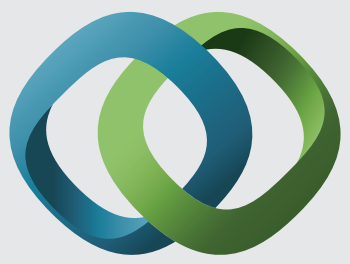

\section{Hindawi}

Submit your manuscripts at

http://www.hindawi.com
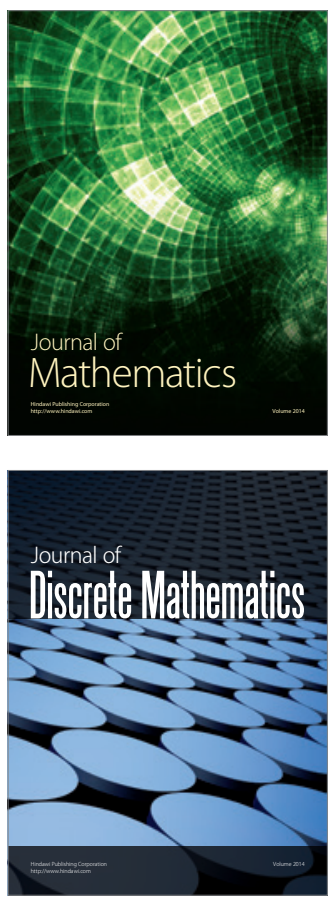

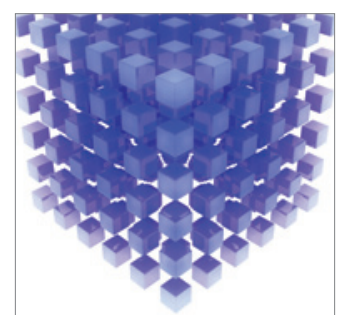

Mathematical Problems in Engineering
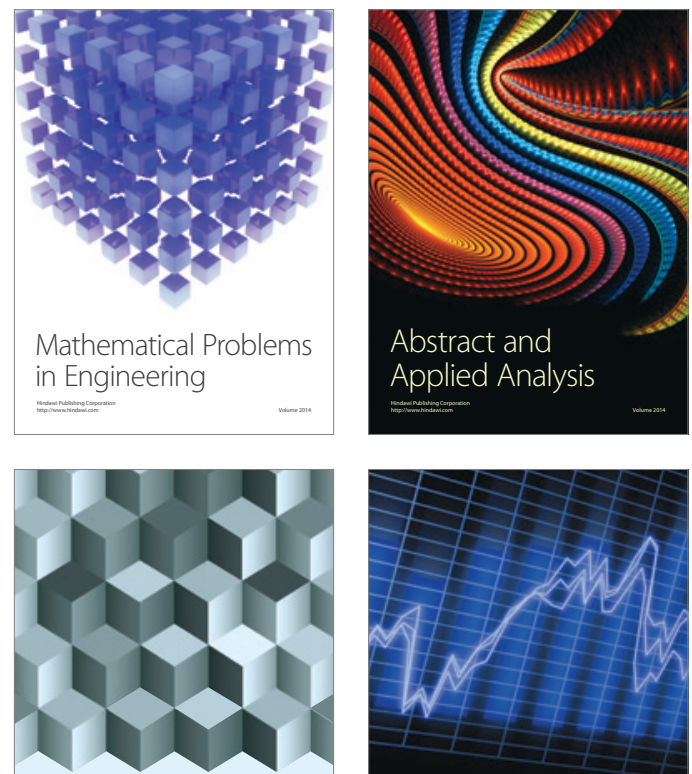

Journal of

Function Spaces

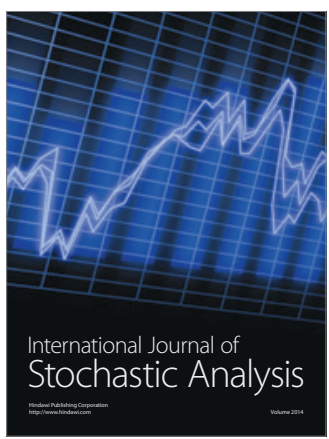

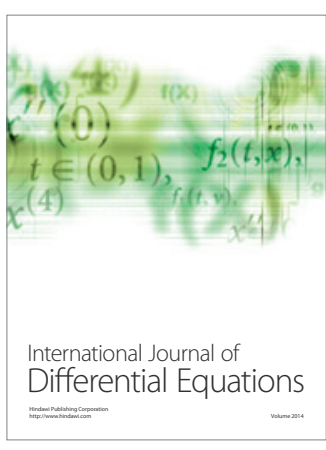
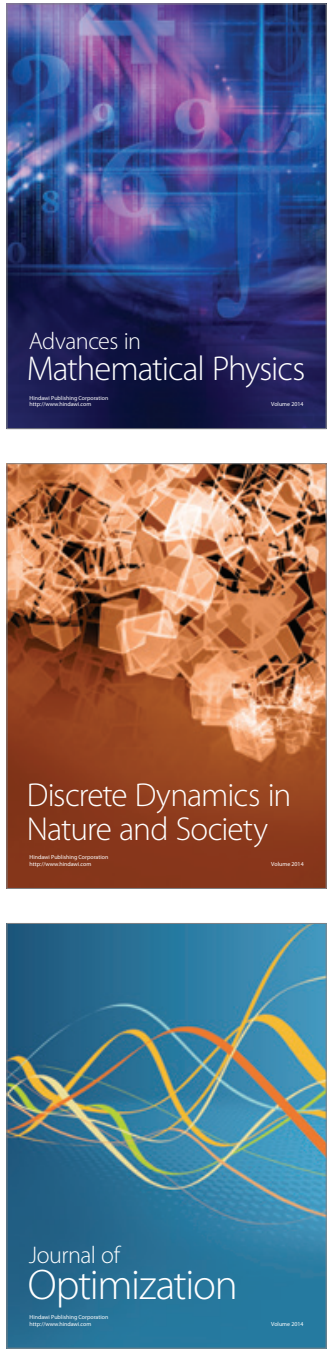